\title{
Effects of Raising and Sueding on the Physical and Mechanical Properties of Dyed Knitted Fabric
}

\section{Md. Touhiduzzaman ${ }^{1}$, Rashid KMM² and Md. Syduzzaman ${ }^{3 *}$}

${ }^{1}$ Department of Fabric Manufacturing Engineering, Bangladesh University of Textiles, Dhaka, Bangladesh

${ }^{2}$ Department of Yarn Manufacturing Engineering, Bangladesh University of Textiles, Dhaka, Bangladesh

${ }^{3}$ Department of Textile Engineering Management, Bangladesh University of Textiles, Dhaka, Bangladesh

\begin{abstract}
For developing finishing methods and broadening their assortment, the research has explored raising and sueding methods in order to find out how these two finishing processes affect the aesthetic, dimensional and functional properties of dyed knitted fabric. In this work, three types of sample fabrics namely three thread fleece or Chief Value Cotton (CVC) fleece, two thread terry or organic fleece and $2 \times 2$ rib were used. CVC fleece was produced from yarns of count $30 \mathrm{Ne}$ and $20 \mathrm{Ne}$ respectively. Organic fleece and $2 \times 2$ rib were produced from $100 \%$ cotton. In this task, we studied six aspects of test results viz. the GSM test, shrinkage test, bursting strength test, spirality test, pilling test and Color fastness to wash. In addition, Wales per Inch (WPI), Course per Inch (CPI), stitch length, stitch density, course length was taken into consideration. The testing procedures were performed meticulously under ideal testing standards. The test results affirm significant effects regarding GSM, shrinkage, bursting and spirality test. No variation of pilling and color fastness of samples subjected to raising and seuding was noted.
\end{abstract}

Keywords: Knitted fabric; Raising; Sueding; Bursting strength; Spirality; Color fastness; GSM

\section{Introduction}

The final stage, amongst a series of processes in the manufacturing of textile clothing to impart the necessary decorative and functional characteristics in the end product involving both chemical and mechanical treatments is finishing. Finishing is best regarded as the final stage in the embellishment of the fabrics, most of which, as it comes from the loom or knitting machine, having an unattractive appearance, which persists, although to a less extent, even after dyeing or printing. A simple definition of finishing is the sequence of operations, other than scouring, bleaching and coloring, to which fabrics are subjected to after leaving the loom or knitting machine [1]. On the basis of processing technique, finishing can be segmented into two types viz. chemical and mechanical. Some mechanical finishing techniques are calendaring, beetling, embossing, raising sueding, and glazing [2]. This paper endeavors to discuss the two mechanical processes Raising and Sueding.

Raising is the technique whereby a surface effect is produced on the fabric that gives the fabric a brushed or napped appearance. It is achieved by teasing out the individual fibers from the yarns so that they stand proud of the surface [3]. The surface fibers are lifted up by means of sharp teeth imparting hairiness, softness and warmth. A velvety material surface is obtained by loosening a large number of individual fibers from the fabric. Natural teasels or metal cards are used to doing so. Raising gives a purely artificial character to the cloth. These effects are produced by ejecting only one end of the fibers residing on the outer surface of the yarn whereas the other end remains twisted within the thread construction so that the fibers are not completely detached from the thread. The thread receives considerable loss of strength, if the fibers totally separate from the yarn. So material tension needs to be carefully regulated.

Material tension is selected depending on the factors like staple length, fiber fineness, yarn twisting, fabric binding [4,5]. Raising can be performed in three states that include raising in loom state, raising after fabric preparation and raising after dyeing. There are two ways, wet and dry raising processes. Wet raising requires less time and gives laid pile while dry raising gives prominent pile and takes more time [6]. Wet technique requires less mechanical action whereas dry one needs repeated drum roller action. So moisture content of the fabric is also very important [7].

Sueding is technically known as peach finish mostly in woven manufacturing process. It's a mechanical finishing process in which a fabric is grazed on one or both sides to raise or create a fibrous surface. A sueding machine produces a very low pile on the fabric surface. The fabric surface is finished to get a feel of suede leather. Sueding machine can have a single cylinder sanding or multi-cylinder emerizing action. Multi cylinder emirizing is preferred over single cylinder process since it removes knots and loose fibers are not produced [8]. The abrasive material used for sanding or emerizing needs to be changed after certain number of circles of actions for uniform formation of pile. The abrasive grit size is determined by the fabric construction so that an optimum sueding effect can be obtained. Sueding effect can range from mild sueding to peach skin effect. By this action, a low pile is produced. It improves touch thus making the fabric surface softer and voluminous. It is very useful for synthetic material to beget a better textile quality.

Both raising and sueding improve the fabric appearance, give the fabric a softer, fuller hand. Masking of the fabric construction, subduing of coloration are also achieved. These improved aesthetics can increase the value of the fabric in the marketplace. Besides, both raising and sueding affect the dimensional as well as physical properties. At the same time, the processes being mechanical cause fiber damage. So finer count of yarn is used for fabric that requires raising and sueding. An assessment of the objective of raising- sueding can be drawn by studying the properties of raised and sueded fabric in comparison

${ }^{*}$ Corresponding author: Md. Syduzzaman, Department of Textile Engineering Management, Bangladesh University of Textiles, 92 Shaheed Tajuddin Ahmed Ave Dhaka 1208, Bangladesh, Tel: 880-2-9114260; E-mail: sayeed33tex@gmail.com

Received November 04, 2015; Accepted December 01, 2015; Published December 15, 2015

Citation: Touhiduzzaman, Rashid KMM, Syduzzaman (2015) Effects of Raising and Sueding on the Physical and Mechanical Properties of Dyed Knitted Fabric. $J$ Textile Sci Eng 5: 228. doi:10.4172/2165-8064.1000228

Copyright: (c) 2015 Touhiduzzaman, et al. This is an open-access article distributed under the terms of the Creative Commons Attribution License, which permits unrestricted use, distribution, and reproduction in any medium, provided the original author and source are credited. 
Citation: Touhiduzzaman, Rashid KMM, Syduzzaman (2015) Effects of Raising and Sueding on the Physical and Mechanical Properties of Dyed Knitted Fabric. J Textile Sci Eng 5: 228. doi:10.4172/2165-8064.1000228

with only dyed fabric. Fabrics of same structure at different processing stages show different values for GSM, bursting strength, pilling test, spirality and shrinkage upon implementing raising- sueding.

\section{Materials and Methods}

Three types of fabric samples such as three thread fleece or CVC fleece, two thread terry or organic fleece, $2 \times 2$ rib were taken to analyze. CVC fleece was made from yarn of count $30 \mathrm{Ne}$ and 20 Ne. CVC Fleece or 3-thread fleece was produced from ring spun yarn in circular knitting machine (Machine gauge 20, Machine dia 30"). Organic Fleece or 2-thread fleece was produced from ring spun yarn in circular knitting machine (Machine gauge 24, Machine dia 30"). 2 $\times 2$ Lycra rib was produced from ring spun yarn in circular knitting machine (Machine gauge 20, Machine dia 32").

CP Fleece or Single Action or European Style, "Normal" double drum machine has been used for CVC Fleece, organic Fleece and 2 $\times 2$ Lycra rib. Here, European style- MC20/24 GF has been followed. As all type of fillets are not suitable for all type of fabrics, we had to be very careful to choose the right type of fillet for the right type of fabric. For CVC fleece fillet types were: Lower drum: 28/32 rubber-pile/ counterpile and upper drum: wire 22 felt- 3 counterpile/ 1 mushroom. For organic fleece or $100 \%$ cotton fleece fillet types were: Lower drum-wire 22 felt-pile/counterpile and upper drum-wire 22 felt-3 counterpile/1 mushroom. Lisa 4 Knit Plus machine has been used for getting sueding or peach effect on organic fleece and $2 \times 2$ Lycra rib (Table 1).

\section{Test method}

Wet raising process was done to avoid higher damage of yarn. Sueding was carried out with multi cylinder option. The standards that were followed in performing the tests are:

\begin{tabular}{|l|l|}
\hline Fabric weight (GSM) & EN 12127 \\
\hline Pilling test & ISO 12945-1 \\
\hline Bursting strength test & ISO 13938-2 \\
\hline Spirality test & In House Method (1 wash) \\
\hline Shrinkage test & In House Method (1 wash) \\
\hline Color fastness to washing & ISO 105 C06 \\
\hline
\end{tabular}

\section{Results and Discussions}

\section{Visual appearance}

The appearance of the samples when dyed and undergone raising and sueding appears prominently distinguishable to the naked eye.

From the Figures 1, 3, 5 and 7 we can see that the gray and dyed samples have distinctively visible intermeshing points with the loops clearly distinguishable. Then, in the Figures 2, 4 and 6 the distinction is replaced by a hairy surface with erect protruding fibres. It is also viewed that the raising process has abraded the surface more intensely than sueding.

\begin{tabular}{|l|c|l|l|}
\hline Fabric type & $\begin{array}{c}\text { Stitch length } \\
(\mathbf{m m})\end{array}$ & Final color & Processing \\
\hline $\begin{array}{l}\text { CVC fleece or } \\
\text { 3-thread fleece }\end{array}$ & 4.46 & Medium grey & $\begin{array}{l}\text { Face side: None } \\
\text { Back side: Raising }\end{array}$ \\
\hline $\begin{array}{l}\text { Organic fleece or } \\
\text { 2-thread terry }\end{array}$ & 2.27 & Real black & $\begin{array}{l}\text { Face side: Sueding } \\
\text { Back side: Raising }\end{array}$ \\
\hline 2x2 lycra rib & 3.20 & Real black & $\begin{array}{l}\text { Face side: Sueding } \\
\text { Back side: Sueding }\end{array}$ \\
\hline
\end{tabular}

Table 1: Processing parameters of samples.

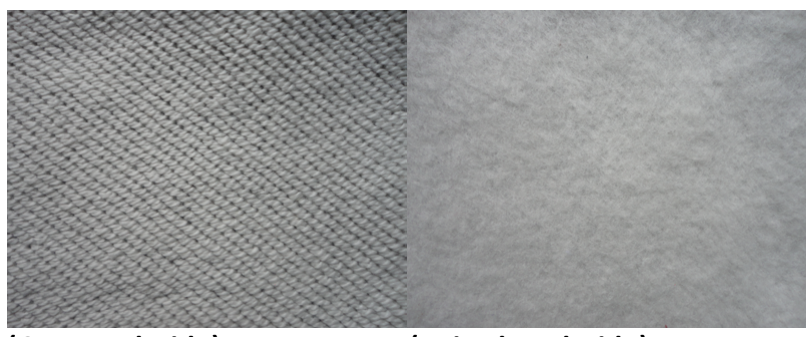

(Gray-Back side) (Raised-Back side)

Figure 1: CVC Fleece.

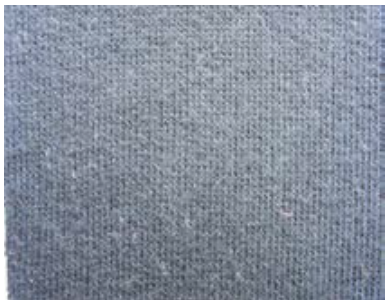

(Dyed-face side)

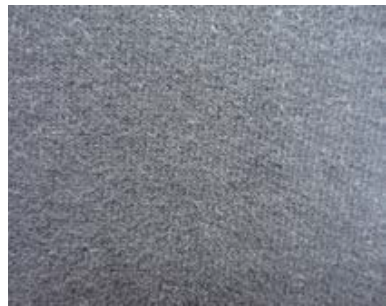

(sueded-face side)

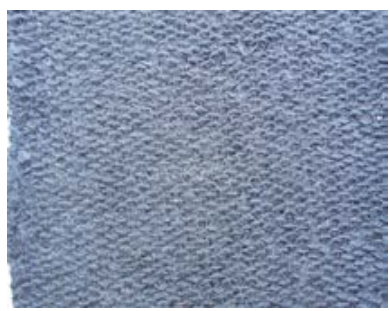

(Dyed-Back side)

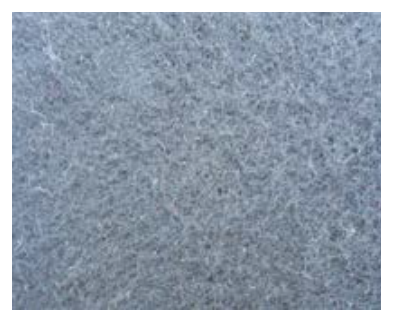

(Raised-back side)

Figure 2: Organic Fleece.

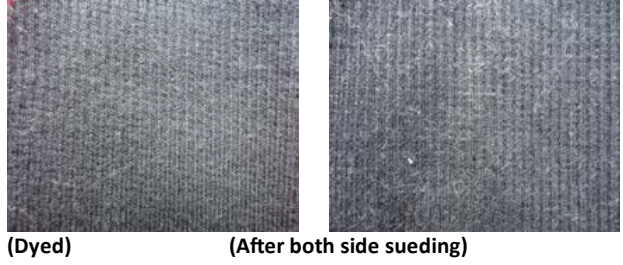

Figure 3: 2 × 2 Lycra rib.

\section{Characteristic tests}

The behavior of the samples under testing restraints allows us to characterize them with respect to bulk, softness, shrinkage etc. thus providing the scope to cast comments. Six tests have been used to bring forth the effects of raising and sueding. These are: Fabric weight (GSM), Pilling test, Bursting strength test, Shrinkage test, Spirality test, Color fastness to washing.

\section{Fabric weight (GSM)}

The weight of a fabric can be described in two ways, either as the 'weight per unit area' or the 'weight per unit length'. If either one of the two or the width of the cloth is known, the other can be calculated assuming the selvedge to have a negligible effect $[9,10]$. We opted for 


\section{GSM}

\section{Grey $\quad$ After dyeing $\quad$ After finishing}

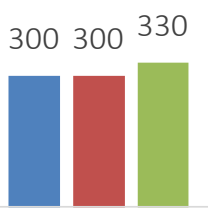

CVC fleece

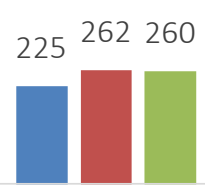

Organic fleece

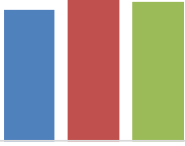

$2 * 2$ lycra rib

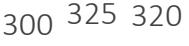

Figure 4: Effect of Raising and sueding on the property of fabric weight.

\section{Bursting pressure Bursting Height}

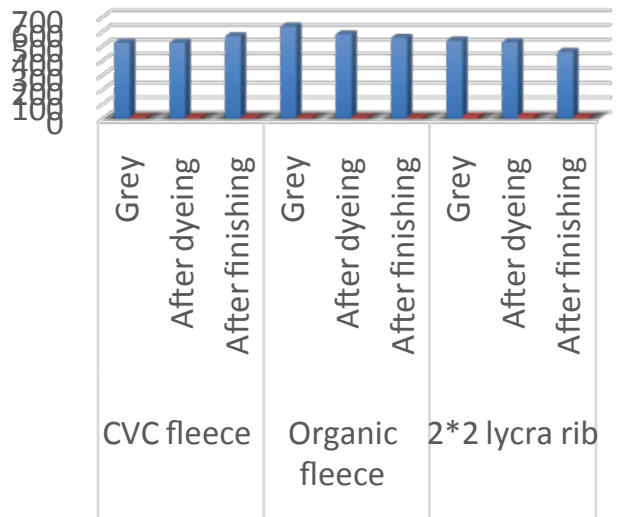

Figure 5: Bursting strength and height of samples.

GSM (Gram per Square Meter) for convenient understanding. EN 12127 method was used in this case. Five samples were taken by using GSM cutter. Then they were weighted on the electric balance. GSM of different stages of processing with and without raising- sueding has been calculated. From those data the average value was calculated.

CVC fleece was produced from dyed yarn. Since the fabric was not dyed further, the gray and dyed state refer to the same condition. So there is no variation in GSM in these states. When dyeing is performed to the yarn, flying fibers reduce, strength and mass of yarn is increased, the twisted fibers become more compact which leads to less raising action. As a result, increase in mass due to dye particles is more than decrease in mass due to fiber loss during raising. So GSM increased after finishing (raising) though there is fibre loss on the surface. Again, in case of organic fleece and Lycra rib, GSM increased notably after dyeing. Because of the absorption of the dye particles, the air and space in between the fibres, loops were replaced. The mass of dye directly contributed to the augmented GSM. Due to Finishing (raising or sueding or both), decrease in mass due to loss of fiber was more than increase of mass due to dye material.

\section{Bursting strength test}

The distending force applied at right angles to the plane of the fabric, under specified condition, which results in the rupture of a textile material is known as bursting strength [11]. Two parameters, bursting pressure (in $\mathrm{KPa}$ ) and bursting height (in $\mathrm{mm}$ ) are attributed to the measure of strength. To do this, force is applied for a duration of $20 \pm 3$ seconds such that the material ruptures within this time. ISO 13938-2 standard was followed here.

\section{Machine parameter for the test}

\begin{tabular}{|c|c|}
\hline Test Area & $50 \mathrm{~cm}$ \\
\hline Clamping & $6.0 \mathrm{Bar}$ \\
\hline Pressure Rate & $10 \mathrm{kPa} / \mathrm{s}$ \\
\hline Pressure Drop & $10 \mathrm{kPa}$ \\
\hline Diaphragm & $1.0 \mathrm{~mm} \mathrm{Duraflex}$ \\
\hline Correction rate & $2 \mathrm{kPa} / \mathrm{s}$ \\
\hline Correction & $35.2 \mathrm{kPa} @ 46.1 \mathrm{~mm}$ \\
\hline
\end{tabular}

It is evident that CVC fleece experienced an increased pressure to rupture but its height has reduced. Yarn dyed fabric has more prominent loops. There is no accumulation in between the adjacent threads. After raising, ejected fibers took stand and the space between the adjacent threads was closed. So, higher pressure was needed to rupture the finished (raised) fabric. Organic fleece shows a gradual reduction in pressure but its bursting height increased after dyeing. When raised and sueded, the bursting pressure reduced because of the fabric becoming weaker. When the gray fabric was dyed, adjacent threads became more compact. After finishing, this compactness was opened. Due to the open end fibers, strength was reduced. In all these cases, duration of 20 seconds could be maintained. As we approach to Lycra rib, the reduction in bursting pressure after sueding is gigantic and the fabric ruptures long before 20 seconds is reached. Lycra is a highly extensible material. As it passes through various rollers and drums for sueding, its strength is reduced.

\section{Shrinkage test}

Shrinkage is the linear amount a fabric will contract warp wise (along wales) or filling wise (along course) when laundered. It is expressed as a percent of its original measurement. All fibers have some tendency to shrink, but this tendency is greatly increased if the fabric has been stretched in finishing [12]. Shrinkage is the process in which a fabric becomes smaller or extended than its original size, usually through the process of laundry. Cotton fabric suffers from two main disadvantages of shrinking and creasing during subsequent washing. When the fabric becomes smaller it is called negative shrinkage, in other case it is termed as positive shrinkage. Shrinkage is mainly due to yarn swelling and the resulting crimp increase. The largest amount of shrinkage is that represented by increase of crimp; yarn shrinkage takes a second place, being generally much less than increase in crimp, while fibre shrinkage is almost negligible [13]. Shrinkage is measured in two ways such as lengthwise and widthwise. For shrinkage test, $50 \mathrm{~cm} \times 50$ $\mathrm{cm}$ sample of each type of fabric was taken. In House Method (1 wash) resulted in the following measurements (Figure 6).

The CVC fleece shows major widthwise expansion whereas the organic fleece and lycra rib went through major lengthwise shrinkage. Since the processes of raising and sueding are mechanical and employ the fabric to be passed through rollers, dimensional integrity is compromised.

\section{Spiraliy test}

"Spirality" is a dimensional distortion in circular knitted fabric that arises from twist stress in the constituent yarns of plain fabric causing all loops to distort and throwing the fabric wales and courses into an 
Citation: Touhiduzzaman, Rashid KMM, Syduzzaman (2015) Effects of Raising and Sueding on the Physical and Mechanical Properties of Dyed Knitted Fabric. J Textile Sci Eng 5: 228. doi:10.4172/2165-8064.1000228

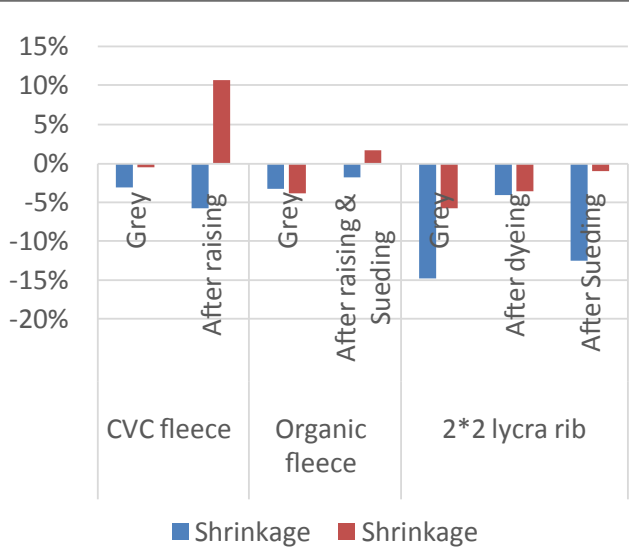

Figure 6: Shrinkage \% along length and width of samples.

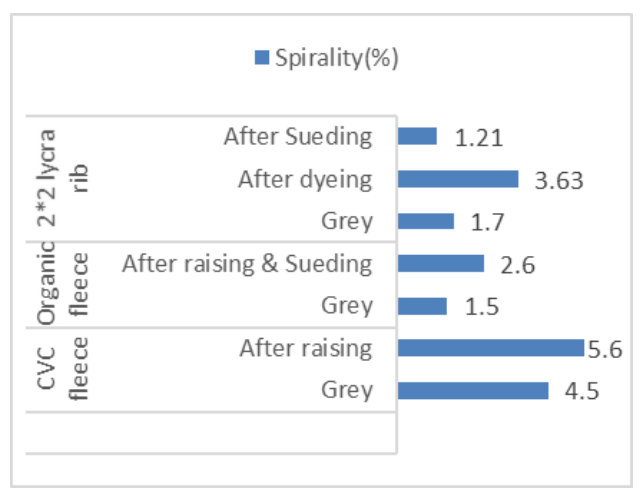

Figure 7: Spirality at different sample state.

angular relationship other than 90 degree. In House Method (1 wash) is used here. The length of left and right seam is measured and the average value for precision result is calculated. Now the distortion of the widths of the bag at the open end is measured. It is done at both end and calculated the average for the precision of the value. Now the spirality can be measured from the following equation: (Figure 7).

\section{Spirality $=($ Distortion $/$ length $) \times 100 \%$}

Both CVC and organic fleeces experienced increased spirality. Hence, dimensional stability and retaining its shape when worn are hampered. Lycra rib confronted more than double spirality after dyeing. After sueding, it was reduced again. This behavior is due to the presence of Lycra in the fabric.

\section{Pilling test}

Pilling is a fabric-surface fault characterized by little 'pills' of entangled fibre clinging to the cloth surface and giving the garment an unsightly appearance. The pills are formed during wear and washing by the entanglement of loose fibres which protrude from the fabric surface. After rubbing of a fabric it is possible to assess the amount of pilling quantitatively either by counting the number of pills or by removing and weighing them. Counting the pills and/or weighing them as a measure of pilling is very time consuming and there is also the difficulty of deciding which surface disturbances constitute pills. The more usual way of evaluation is to assess the pilling subjectively by comparing it with either standard samples or with photographs of them or by the use of a written scale of severity. ISO 12945-1 was used as reference in this case.

\section{Pilling scale}

The pilling scale was explained at Table 2 .

\section{Pilling result}

Severe pilling occurred in every case. As raising and sueding cause fibres to be raised and ejected, the free ends of the fibres can easily entangle to form pills. In case of $2 \times 2$ Lycra rib it showed exceptionality. Dyed rib fabric showed severe pilling but sueding affected that same fabric to show slight pilling (Table 3 ).

\section{Color fastness test}

Color fastness to wash is achieved by placing a specimen of textile, in contact with one or two specific adjacent fabrics and is mechanically agitated under described conditions of time and temperature in a soap solution, then rinsed and dried. The change in color of the specimen and the staining of the adjacent fabric are assessed with the grey scales. According to ISO 105 C06, DW multi-fibre fabric was used to recognize any kind of staining or color deterioration.

\section{Grey scale for color change and staining}

The grey scale for color change and staining was clearly explained at Table 4 .

\section{Color fastness result}

Color fastness to wash depends on the selection of raw materials, dyes and chemicals. According to our experiments it can be said that the type of finishing process such as raising, sueding don't give any impact on coloration of the fabric (Table 5).

\begin{tabular}{|c|c|}
\hline Rating & Surface evaluation \\
\hline 5 & No pilling \\
\hline 4 & Slight pilling \\
\hline 3 & Moderate pilling \\
\hline 2 & Severe pilling \\
\hline 1 & Very severe pilling \\
\hline
\end{tabular}

Table 2: Pilling Scale.

\begin{tabular}{|l|l|c|c|}
\hline Fabric Type & \multicolumn{2}{|l|}{ Grading Result } & Remarks \\
\hline \multirow{3}{*}{ CVC fleece } & Grey & 2 & Severe pilling \\
\cline { 2 - 4 } & After finishing & 2 & Severe pilling \\
\hline \multirow{3}{*}{ Organic fleece } & Grey & - & \\
\cline { 2 - 4 } & After dyeing & 2 & Severe pilling \\
\cline { 2 - 4 } & After finishing & 2 & Severe pilling \\
\hline \multirow{3}{*}{$2 \times 2$ lycra rib } & Grey & - & Severe pilling \\
\cline { 2 - 4 } & After dyeing & 2 & Slight pilling \\
\hline & After finishing & 4 & \\
\hline
\end{tabular}

Table 3: Pilling Result.

\begin{tabular}{|c|c|}
\hline 1 & 3 \\
\hline $1 / 2$ & $3 / 4$ \\
\hline 2 & 4 \\
\hline $2 / 3$ & $4 / 5$ \\
\hline 3 & 5 \\
\hline
\end{tabular}

Here, $5=$ Best rating refers to no color change as well as no staining. Table 4: Gray Scale. 
Citation: Touhiduzzaman, Rashid KMM, Syduzzaman (2015) Effects of Raising and Sueding on the Physical and Mechanical Properties of Dyed Knitted Fabric. J Textile Sci Eng 5: 228. doi:10.4172/2165-8064.1000228

Page 5 of 5

\begin{tabular}{|l|l|c|c|}
\hline Fabric Type & Color change & Staining \\
\hline CVC fleece & Grey & $4 / 5$ & $4 / 5$ \\
\cline { 2 - 4 } & After raising & $4 / 5$ & $4 / 5$ \\
\hline \multirow{3}{*}{ Organic fleece } & Grey & $4 / 5$ & $4 / 5$ \\
\cline { 2 - 4 } & After dyeing & $4 / 5$ & $4 / 5$ \\
\cline { 2 - 4 } & After raising and Sueding & $4 / 5$ & $4 / 5$ \\
\hline \multirow{3}{*}{ 2x2 lycra rib } & Grey & $4 / 5$ & $4 / 5$ \\
\cline { 2 - 4 } & After dyeing & $4 / 5$ & $4 / 5$ \\
\cline { 2 - 4 } & After Sueding & $4 / 5$ & $4 / 5$ \\
\hline
\end{tabular}

Table 5: Color fastness result.

\section{Conclusion}

It was observed that the appearance of the samples was directly affected. GSM was also found to be varied thus fill and volume of the fabric was changed. GSM of CVC fleece was increased but the other two samples saw the opposite consequence. Strength test elucidated that the samples lost strength to some extent. $2 \times 2$ Lycra rib was found to be heavily affected regarding strength. Shrinkage and spirality illustrated that the dimensional integrity was compromised whereas $2 \times 2$ Lycra rib suffered the most variation. CVC and organic fleeces resulted in a widthwise expansion. CVC and organic fleeces were found to be highly prone to pilling but $2 \times 2$ Lycra rib presented slight pilling. Color fastness to wash showed no impact. So fabrics could be raised and sueded without the concern of coloration and color fastness to wash being hampered.

\section{References}

1. Marsh JT (1966) An Introduction to Textile Finishing. $2^{\text {nd }}$ eds. Open library, London.

2. Shenai VA (1990) Technology of Textile Finishing.

3. Palmar JW (1996) Textile Processing and Finishing Aids: Recent Advancements.

4. Rouette HK (2011) Encyclopedia of Textile Finishing Volume III.

5. Arora SM (1983) Modern Techniques of Textile Dyeing Bleaching and Finishing Small Industry Research Institute.

6. Murphy WS (2000) Technology of Textile Finishing

7. Haigh D (1971) Dyeing and Finishing Knitted Goods. Hosiery Trade Journal, England.

8. Manual of Raising, Shearing and Sueding of Mario Crosta.

9. Booth JE (1969) Principles of Textile Testing. Chemical Publishing, New York USA.

10. Skinkle JH (1972) Textile Testing: Physical, Chemical and Microscopical. Chemical Pub Co, New York, USA.

11. ASTM International.

12. Johnson GH (1927) Textile fabrics: Their selection and care from the standpoint of use, wear, and launderability. Harper \& Brothers, New York, USA.

13. Collins GE (2009) Fundamental principles that govern the shrinkage of cotton goods by washing. Journal of the Textile Institute Proceedings 30: 46-61. 